\title{
In This Issue: August 2021
}

\section{Adam M. Brenner ${ }^{1}$ (D)}

Published online: 6 July 2021

(C) Academic Psychiatry 2021

The August 2021 issue of Academic Psychiatry offers valuable papers that address how psychiatry residents are doing, but in very different senses of the question. First, have they demonstrated competence? And second, how are they holding up under the strain of training in psychiatry?

Recently, Academic Psychiatry editors discussed the problematic aspects of the Clinical Skills Evaluation (CSE) and concluded by calling for more rigorous studies of the process and its outcomes [1]. I am pleased to report that Academic Psychiatry has two papers in this issue that significantly advance the understanding of the reliability and the validity of the examination $[2,3]$. Both studies were led by Michael D. Jibson, M.D., Ph.D., who has been a pivotal leader in the development and assessment of tools for residency programs to use in fulfilling this requirement for certification by the American Board of Psychiatry and Neurology. (Disclosure: Dr. Jibson currently serves on the Academic Psychiatry Governance Committee as the representative of the Association for Academic Psychiatry, one of the journal's sponsoring organizations.)

Jibson and Keshavarzi [2] report on the CSE's reliability. Although the CSE is designed to be administered as part of residents' progress through training, a pool of graduates has also been seeking opportunities to pass the exam. Between 2012 and 2017, the University of Michigan administered the CSE 159 times to 51 such candidates. Thirty-six of these interviews were rated by two evaluators. How did they do in interrater reliability? The CSE covers three distinct skills. For rating of the physician-patient relationship, the intraclass correlation was at the upper end of fair, while for conduct of the interview and case presentation, the correlation rose to the good range [4].

Interestingly, the correlation was not uniform across the scoring range. Evaluators had far more consistency in

Adam M. Brenner

Adam.Brenner@utsouthwestern.edu

1 University of Texas Southwestern Medical Center, Dallas, TX, USA agreeing on passing exams than failing exams. The authors argue that this finding is consistent with the intent of the American Board of Psychiatry and Neurology to move away from a high-stakes exam, where the emphasis is on screening out unacceptable physicians, to an iterative process meant to reinforce and develop competency. Nonetheless, Jibson and Keshavarzi rightly observe that there is room for improvement in "the identification of inadequate performance" [2] and suggest several avenues to improving consistency between evaluators and between programs. This is an important point. It is precisely in identifying unacceptable performance that psychiatric educators should have the most concern about unconscious bias in favor of their program's residents and about the pressure of the residents' and the program's interests on the process.

Test-retest reliability was assessed from the first to the third CSE taken. Here, the ratings for the three skills were consistently in the higher end of the good range. Significant improvement was also demonstrated, with a moderate effect size. Graduate examinees improved with practice and feedback from evaluators over the course of the day. It is hard to tell how much this finding represents learning the test versus actual growth in skills. It is also hard to know how this finding would carry over to residents when CSEs are separated by weeks or months. Yet, overall, this paper increases confidence in the reliability of the CSE while also suggesting directions for future study.

In a separate paper [3], Jibson et al. examined validity of the CSE through analysis of 1,156 examination results at four residency programs. The authors used the unitary framework of validity as their guide. This framework approaches validity from multiple angles, looking at content, response process, internal structure, association with other variables, and consequences. This study, however, was limited to the dimensions of response process and association with the variables of time in program and scores from the Psychiatry Resident-InTraining Examination (PRITE).

As hypothesized, the association with time in training was found to be quite strong for all three skills, but especially so for interview scores. By contrast, the CSE had very low 
correlation with PRITE scores, also as hypothesized. There is no reason to think that interviewing or presentation skills should correspond with success on multiple-choice tests of medical knowledge.

The results seem more complicated with regard to "response process," which the authors use to refer to the patterns of scoring across programs and across faculty. The authors note that most of the difference in scoring pattern was "attributable to a broad range of scores early in residency; by PGY-4 [postgraduate year 4], there was only a $6 \%$ difference in pass rates among the programs. In fact, the programs had comparable pass rates overall" [3]. But it is worth looking more closely at the pattern, particularly that the passing rate among the programs had "a range of 39-96\% in PGY-1" [3]. It is hard to know what to make of a program finding that $96 \%$ of its CSE assessments in postgraduate year 1 demonstrated the performance expected of a competent practicing psychiatrist. This finding suggests the possibility of using exam data patterns to identify sites whose use of the exam raises questions of "face validity."

As hoped, the authors did find a reasonable range of scores by the faculty, although the faculty tended to avoid using the highest or lowest ends of the scale. They also found that on average, residents required more than three attempts to meet the required target of three passed exams (with a significant minority requiring five or more attempts), lending support to the idea that programs were not simply approving residents routinely. Many residents did not pass all three CSE assessments until postgraduate year 4 . That the pressure on a program to pass all its residents is likely felt primarily in the fourth year slightly mitigates this reassurance.

The authors noted that this study looked only at two of the five elements of the unitary framework of validity. Given that no other exam is available to use as a gold standard, it may not be possible to study the dimension of "content," and the exam may not lend itself to examination of "internal structure." But as a field, psychiatry has a glaring need for studies that look at the consequences to patients and to residents of the use of CSEs. What are the effects on patient outcomes and on professional development? More work needs to be done before any final conclusions are drawn about validity. Nonetheless, the value of Jibson et al.'s multisite study in supporting the validity of the CSE is clear.

Now, we turn our attention from competency to flourishing. Two papers help us think about how to lessen the impact of residency stressors on the growth and wellbeing of trainees.

Herschkopf's commentary [5] is an important contribution to this literature. It brings together concepts from very different professional and intellectual traditions. Moral distress, an idea that has become increasingly prominent in medical education, is rooted in a rich literature of nursing and medical ethics and burnout. Countertransference is a concept with a century's history of use - and confusion - derived from psychoanalytic theory and practice. Many of us will have drawn on these concepts to help our residents when they are distressed (though Herschkopf focuses on residents, the lessons here apply to medical students and faculty as well). But have we thought about why we use one or the other framework? Or what happens when we attempt to apply them together?

These questions do not have simple answers. Herschkopf, however, demonstrates how much value can come from integrating these different frameworks. Once we hold them in mind together, we can be significantly more nuanced in our support of residents. We have all learned the caution "If all you have is a hammer, everything looks like a nail." Having different conceptual tools supports us in diagnosing different kinds of problems. Further to this, as the cases illustrate [5], situations do not sort themselves neatly into the categories of "clinical moral dilemma" versus "emotional reaction to a challenging patient." Many situations will be both and will require a supervisor's flexible consideration of both frameworks of distress.

So, in thinking about trainee stress, we have the conceptual tools of moral distress and countertransference. Padilla et al. [6] remind us to add the concept of "adverse events." A trainee experiencing moral distress or countertransference is often afraid of how the situation will turn out; the language of adverse events is needed once the outcome has in fact been bad. At that point we may shift from a countertransference model (understanding managing disturbing affect), to a trauma model (providing safety and the means to heal from overwhelming experience). We also must use the framework of root cause analysis and risk management to engage the clinical system in change to prevent future adverse events.

Of course, these tasks of support and system change are part of every program director's daily life. Padilla et al. demonstrate an approach to systematizing the reporting and the response by creating an Adverse Events Committee [6]. Their report is a work in progress, with $74 \%$ of residents attesting to the value of the committee but only $41 \%$ believing the program was safer. Perhaps, this finding is not surprising: residency programs generally have more influence on the subjective experience of residents than over the management and change processes at clinical sites.

So, to return to the question of how our residents are doing, like with everything else in psychiatry, it is complicated. The journal is proud to add to and embrace that complexity.

\section{Declarations}

Disclosures The author states that there is no conflict of interest. 


\section{References}

1. Balon R, Beresin EV, Guerrero APS, Morreale MK, Aggarwal R, Louie AK, et al. Clinical skills verification: a problematic examination. Acad Psychiatry. 2020;44:255-9.

2. Jibson MD, Keshavarzi N Psychiatry clinical skills evaluation: interrater reliability of the American Board of Psychiatry and Neurology required assessment. Acad Psychiatry. 2020. Available https://doi.org/10.1007/s40596-020-01337-3. Last accessed 21 June 2021

3. Jibson MD, Agarwal G, Anzia JM, Summers RF, Young JQ, Seyfried LS Psychiatry clinical skills evaluation: a multisite study of validity. Acad Psychiatry. 2021. Available at: https://doi.org/10. 1007/s40596-020-01388-6. Last accessed 21 June 2021
4. Cichetti DV. Guidelines, criteria, and rules of thumb for evaluating normed and standardized assessment instruments in psychology. Psychol Assess. 1994;6:284-90.

5. Herschkopf MD. Moral distress or countertransference? Addressing emotional reactions of psychiatry trainees. Acad Psychiatry. 2021. Available at https://doi.org/10.1007/s40596-021-01443-w. Last accessed 21 June 2021

6. Padilla V, Healey J, Salinas J, Bryant C, Muñoz O Durand D. Residents impacted by adverse events: the development of a pilot committee for responding to and supporting psychiatry residents. Acad Psychiatry. 2020. Available at: https://doi.org/10.1007/ s40596-020-01348-0. Last accessed 21 June 2021

Publisher's Note Springer Nature remains neutral with regard to jurisdictional claims in published maps and institutional affiliations. 